\title{
Atividade antimicrobiana de óleos essenciais de condimentos frente a amostras de Escherichia coli isoladas de aves e bovinos
}

\author{
Antimicrobial activity of spice essential oils against Escherichia coli strains isolated from \\ poultry and cattle
}

\begin{abstract}
Deise Flores Santurio ${ }^{*}$ Mateus Matiuzzi da CostaII Grazieli Maboni' ${ }^{\mathrm{III}}$ Carlos Pasqualin Cavalheiro ${ }^{\mathrm{I}}$ Mariangela Facco de SáIII Marcelo Dal Pozzo $^{\text {III }}$ Sydney Hartz Alves ${ }^{\text {III }}$ Leadir Lucy Martins Fries ${ }^{\mathrm{I}}$
\end{abstract}

\section{RESUMO}

A discutida questão da substituição do uso de antibacterianos em rações (promotores de crescimento) requer urgentes alternativas. Face às necessidades de inibidores microbianos nesses alimentos, os óleos essenciais (OES) se constituem em alternativa, sob avaliação. Neste estudo, avaliouse a atividade antimicrobiana dos OES de Origanum vulgare (orégano), Thymus vulgaris (tomilho), Cinnamomum zeylanicum (canela), Lippia graveolens (orégano mexicano), Zingiber officinale (gengibre), Salvia officinalis (sálvia), Rosmarinus officinalis (alecrim) e Ocimum basilicum (manjericão) frente a amostras de Escherichia coli isoladas de fezes de aves $(n=43)$ e de bovinos $(n=36)$. A concentração inibitória mínima (CIM) e a concentração bactericida mínima (CBM) foram determinadas para cada isolado através da técnica de microdiluição em caldo, a partir da máxima concentração de $6400 \mu \mathrm{g} \mathrm{mL}^{-1}$ de cada OE testado. Observouse atividade antimicrobiana para os OES de orégano, orégano mexicano, tomilho, canela. Para todas as amostras testadas, independente de sua origem, os OES mais e menos efetivos quanto à atividade antimicrobiana foram o orégano e a canela, respectivamente. Esses resultados confirmaram o potencial antibacteriano de alguns $O E S$, os quais merecem novas investigações abordando sua adição na alimentação de aves $e$ bovinos.

Palavras-chave: Escherichia coli, óleos essenciais, atividade antimicrobiana.

\section{ABSTRACT}

The discussed issue about replacing the use of antibiotics in animal feed (growth promoters) requires emerging alternatives. To meet the needs of microbial inhibitors in these foods, the essentials oils (EOS) constitute potential alternatives under evaluation. In this study it was evaluated the antimicrobial activities of EOs from Oreganum vulgare (oregano), Thymus vulgaris (thyme), Lippia graveolens (Mexican oregano), Cinnamomum zeylanicum (cinnamon), Zingiber officinale (ginger), Salvia officinalis (sage), Rosmarinus officinalis (rosemary) and Ocimum basilicum (basil) against Escherichia coli strains isolated from poultry $(n=43)$ and cattle faeces $(n=36)$. The minimum inhibitory concentration (MIC) and minimum bactericidal concentration $(M B C)$ were determined for each isolate by using the broth microdilution technique, from the maximum concentration of $6400 \mu \mathrm{g} \mathrm{mL}^{-1}$ of each OE tested. Antimicrobial activity was observed on the essential oils of oregano, mexican oregano, thymus and cinnamon. For all strains tested, regardless of their origin, the OES more and less effective as antimicrobial activity were oregano and cinnamon, respectively. These results confirm the antimicrobial potential of some EOs, which deserve further research, addressing the addition of essential oils in poultry and cattle feeding.

Key words: Escherichia coli, essential oils, antimicrobial activity.

\section{INTRODUÇÃO}

Escherichia coli é a espécie predominante entre as bactérias intestinais anaeróbicas facultativas e não esporuladas, pertencendo à família Enterobacteriaceae, sendo um bacilo Gram-negativo fermentador da glicose e lactose com produção de ácido

'Departamento de Ciência e Tecnologia dos Alimentos, Centro de Ciências Rurais (CCR), Universidade Federal de Santa Maria (UFSM), Av. Roraima, 1000, Prédio 42, Camobi, 97105-900, Santa Maria, RS, Brasil. E-mail:deisesanturio@ibest.com.br.*Autor para correspondência.

IIFundação Universidade Federal do Vale do São Francisco, Petrolina, PE, Brasil.

IIIDepartamento de microbiologia e parasitologia, Centro de Ciências da Saúde, UFSM, Santa Maria, RS, Brasil. 
e gás, dentre alguns atributos úteis na sua identificação (ITO et al., 2007).

Alimentos de origem animal submetidos a algum processo, como é o caso da carne moída, têm sido identificados como importante veículo de transmissão de $\boldsymbol{E}$. coli do sorotipo O157:H7 e outras STEC (E. coli produtora de toxina Shiga), isoladas de toxinfecções alimentares no homem (GRIFFIN \& TAUXE, 1991). Os bovinos têm sido considerados um importante reservatório de $\boldsymbol{E}$. coli, produtoras da toxina Shiga (SARIDAKIS et al., 1997).

Entre os patógenos bacterianos causadores de infecção em aves, Escherichia coli destaca-se como um dos principais agentes responsáveis por perdas econômicas na indústria avícola. As $\boldsymbol{E}$. coli patogênicas para aves (APEC) representam um conjunto de isolados capazes de determinar doenças extra-intestinais como aerossaculite, pericardite e septicemia (ITO et al., 2007), geralmente denominadas como colibacilose.

A colibacilose pode ser controlada por medidas profiláticas e uso de antibacterianos (McMULLIN, 2004). No entanto, a adição de antibacterianos em doses subclínicas (promotores de crescimento) tem gerado preocupações como a emergência de bactérias resistentes bem como a indesejada presença de seus resíduos em derivados cárneos, leite e ovos (WHO, 2003). Nesse contexto, alternativas substitutivas aos antibacterianos são urgentemente requeridas tais como o uso de óleos essenciais e/ou extratos herbais (BRUGALLI, 2003).

Os Óleos Essenciais (OES), derivados de plantas utilizadas como condimentos, representam complexas misturas de substâncias naturais, tradicionalmente utilizadas para acentuar gosto ou aroma de alguns alimentos. Constituem-se de substâncias, cujos componentes incluem hidrocarbonetos terpênicos, álcoois simples, aldeídos, cetonas, fenóis, ésteres, ácidos orgânicos fixos, em diferentes concentrações, em que um composto farmacologicamente ativo é majoritário (SIMÕES \& SPITZER, 2000). A maior parte da atividade antimicrobiana de OES parece estar associada aos compostos fenólicos (SIMÕES \& SPITZER, 2000). O efeito antimicrobiano está relacionado, principalmente, à alteração da permeabilidade e integridade da membrana celular bacteriana (LAMBERT et al., 2001).

Este estudo teve como objetivos: a) avaliar a atividade antibacteriana in vitro dos óleos essenciais de condimentos Cinnamomum zeylanicum (canela), Lippia graveolens (orégano mexicano), Ocimum basilicum (manjericão), Origanum vulgare (orégano), Rosmarinus officinalis (alecrim), Salvia officinalis (sálvia), Thymus vulgaris (tomilho) e Zingiber officinale (gengibre) sobre amostras de $\boldsymbol{E}$. coli. isoladas de aves e bovinos; b) comparar a atividade dos OES sobre isolados com perfis de susceptibilidade e de resistência a antibacterianos diversos.

\section{MATERIAL E MÉTODOS}

\section{Microrganismos \\ Foram estudadas 79 amostras de} Escherichia coli, sendo 43 isoladas de fezes de aves e 36 de fezes de bovinos, provenientes do Laboratório de Pesquisas Micológicas da Universidade Federal de Santa Maria (UFSM). Todas as amostras foram isoladas de animais sadios.

Teste de suscetibilidade aos antimicrobianos

Os testes de suscetibilidade aos antimicrobianos foram realizados pelo método de difusão em ágar (CLSI M31-A3; 2008) utilizando-se: ampicilina $(10 \mu \mathrm{g})$, sulfametoxazol+trimetoprim $(25 \mu \mathrm{g})$, ceftriaxona $(30 \mu \mathrm{g})$, ciprofloxacina $(5 \mu \mathrm{g})$, gentamicina $(10 \mu \mathrm{g})$, estreptomicina $(10 \mu \mathrm{g})$, ácido nalidíxico $(30 \mu \mathrm{g})$, ceftiofur $(30 \mu \mathrm{g})$, amoxicilina $(10 \mu \mathrm{g})$, amoxicilina+ácido clavulânico $(30 \mu \mathrm{g})$, imipenem $(10 \mu \mathrm{g})$, doxiciclina $(30 \mu \mathrm{g})$, obtidos comercialmente (DME; Araçatuba, São Paulo, Brasil).

Óleos essenciais

Foram utilizados os óleos essenciais de canela, orégano mexicano, manjericão, orégano, alecrim, sálvia, tomilho e gengibre, obtidos comercialmente, em frasco âmbar, lacrados, com volumes de $5 \mathrm{ml}$ ( Essential 7.com; Roewell, New Mexico, USA).

Determinação das concentrações inibitórias mínimas Após pesagem de 1g, cada óleo essencial foi diluído com metanol até atingir a concentração de $640 \mathrm{mg} \mathrm{ml}^{-1}$ (solução I). A seguir, foi diluído na proporção de 1:100 em Caldo Muller-Hinton, obtendose a concentração de $6400 \mu \mathrm{g} \mathrm{ml}^{-1}$ (solução II). Com base no documento M31-A3 do CLSI (2008), volumes de $100 \mu 1$ de caldo Muller-Hinton eram distribuídos nos poços de uma placa de microtitulação. Em seguida, $100 \mu 1$ da solução II eram acrescidos ao primeiro poço e, após homogeneização, transferia-se para o segundo e assim sucessivamente, obtendo-se concentrações finais de 3200, 1600, 800, 400, 200, $100 \mu \mathrm{g} \mathrm{ml}^{-1}$. As colônias de $\boldsymbol{E}$. coli foram desenvolvidas no ágar Muller-Hinton, suspensas em solução salina $0,085 \%$ obtendo-se uma turvação equivalente ao tubo $0,5 \mathrm{da}$ Escala Mac Farland $\left(1 \times 10^{8} \mathrm{UFC} \mathrm{ml}^{-1}\right)$. Desta suspensão, inoculava-se $10 \mu 1\left(1 \times 10^{5} \mathrm{UFC} \mathrm{m}^{-1}\right)$ em cada poço já contendo os óleos essenciais. As microplacas eram incubadas durante $24 \mathrm{~h} / 35^{\circ} \mathrm{C}$, em condições de aerobiose. A CIM consistiu na menor concentração do 
óleo essencial capaz de causar inibição total do crescimento bacteriano. Esses ensaios foram realizados em triplicata.

Determinação das concentrações bactericidas mínimas Definidas como as menores concentrações dos óleos essenciais capazes de causar a morte do inóculo, foram determinadas a partir dos poços onde, após $24 \mathrm{~h}$ de incubação não havia crescimento bacteriano visíveis. Destes transferia-se uma alíquota de $10 \mu 1$ para a superfície do ágar Muller-Hinton. Após $24 \mathrm{~h}$ de incubação a $35^{\circ} \mathrm{C}$, registrava-se a menor concentração dos óleos essenciais, nos quais não se evidenciava crescimento bacteriano. Esses ensaios foram também realizados em triplicatas.

Análise estatística

O teste não paramétrico de Mann-Whitney foi empregado para comparar duas amostras independentes, visando a observar se os diferentes grupos em estudo apresentavam perfis de suscetibilidade semelhantes ou não, frente a determinado óleo essencial.

\section{RESULTADOS}

Os isolados incluídos neste estudo evidenciaram variações na suscetibilidade frente aos diferentes óleos essenciais (OES). As médias geométricas para as Concentrações Inibitórias Mínimas (CIMs) e Concentrações Bactericidas Mínimas (CBMs), frente a $\boldsymbol{E}$. coli isoladas de bovinos foram: Origanum vulgare $\left[\mathrm{CIM}=831,4 \mu \mathrm{g} \mathrm{ml}^{-1}\right.$ e $\left.\mathrm{CBM}=915,4 \mu \mathrm{g} \mathrm{ml}^{-1}\right]$,
Lippia graveolens $\left[\mathrm{CIM}=1131,4 \mu \mathrm{g} \quad \mathrm{ml}^{-1} \quad \mathrm{e}\right.$ $\left.\mathrm{CBM}=1345,4 \mu \mathrm{g} \mathrm{ml}^{-1}\right]$, Thymus vulgaris $[\mathrm{CIM}=2095,1 \mu \mathrm{g}$ $\mathrm{ml}^{-1}$ e CBM $=2262,7 \mu \mathrm{g} \mathrm{ml}^{-1}$, Cinnamomun zeylanicum $\left[\mathrm{CIM}=4354,5 \mu \mathrm{g} \mathrm{ml}^{-1}\right.$ e CBM $\left.=5486,4 \mu \mathrm{g} \mathrm{ml}^{-1}\right]$ e frente $\boldsymbol{E}$. coli isolados de aves foram Origanum vulgare $\left[\mathrm{CIM}=750,1 \mu \mathrm{g} \mathrm{ml}^{-1}\right.$ e CBM=895,5 $\left.\mathrm{g} \mathrm{ml}^{-1}\right]$, Lippia

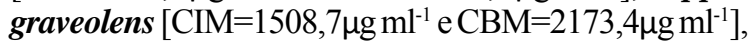
Thymus vulgaris $\left[\mathrm{CIM}=1941,5 \mu \mathrm{g} \mathrm{ml}^{-1} \mathrm{eCBM}=2005,1 \mu \mathrm{g}\right.$ $\mathrm{ml}^{-1}$ ] Cinnamomun zeylanicum $\left[\mathrm{CIM}=3098,5 \mu \mathrm{g} \mathrm{ml}^{-1} \mathrm{e}\right.$ $\left.\mathrm{CBM}=4075,3 \mu \mathrm{g} \mathrm{ml}^{-1}\right]$. No presente estudo, os OES de gengibre, manjericão, alecrim e sálvia não evidenciaram atividade antibacteriana frente aos microrganismos avaliados. A média geométrica das CIMs indicou que o OE de orégano foi o mais ativo para os isolados de aves do que de bovinos $(\mathrm{P}<0,0001)$. Os $\mathrm{OE}$ de orégano mexicano e tomilho foram igualmente ativos para os isolados de aves, enquanto, para os isolados de bovinos, o OE de orégano mexicano foi mais ativo que o OE de tomilho $(\mathrm{P}<0,0001)$. O OE de canela foi menos ativo $(\mathrm{P}<0,0001)$ que os OES de orégano, orégano mexicano e tomilho para os respectivos isolados (Tabela1).

Com base na resistência aos antimicrobianos, as amostras isoladas de aves foram distribuídas em 16 subgrupos e as de bovinos em 11 subgrupos (Tabela 2). A seguir, avaliou-se a suscetibilidade de cada subgrupo frente aos OES e não foram detectadas diferenças significativas nas suscetibilidades desses subgrupos.

\section{DISCUSSÃO}

Desde 2006, a União Européia, importante cliente do mercado brasileiro de carne, baniu o uso de

Tabela 1 - Atividade antimicrobiana $\left(\mu \mathrm{g} \mathrm{ml} \mathrm{ml}^{-1}\right)$ dos óleos essenciais de Origanum vulgare, Lippia graveolens, Thymus vulgaris e Cinnamomum zeylanicum frente a $\boldsymbol{E}$. coli isoladas de aves e bovinos.

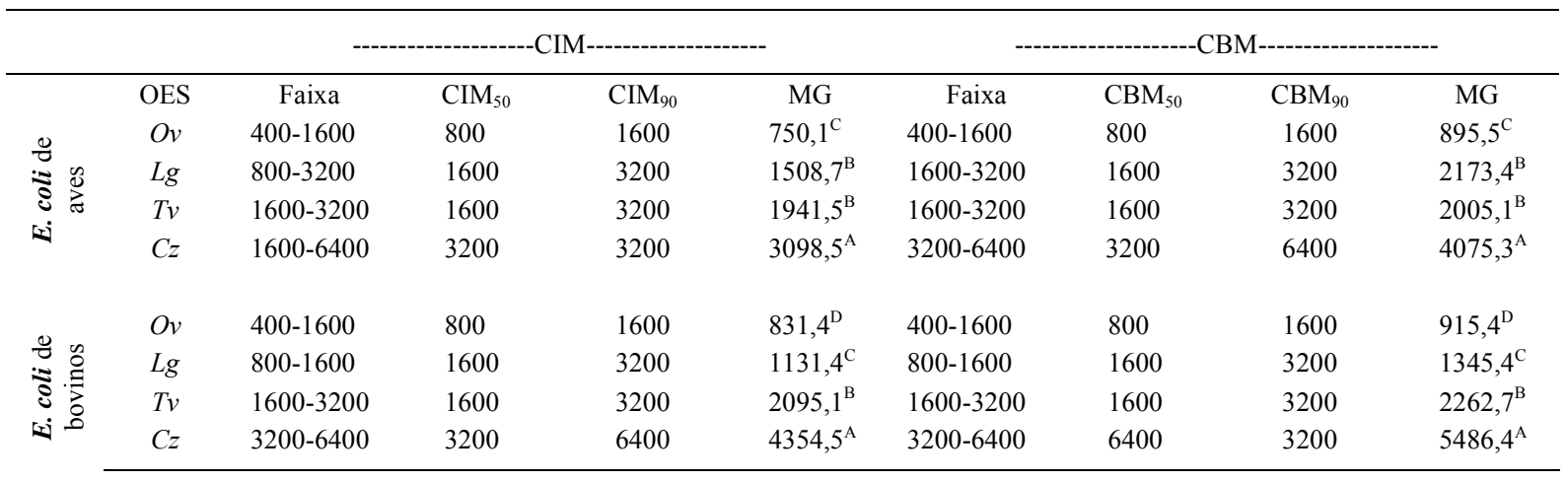

$\mathrm{CIM}=$ Concentração Inibitória Mínima; $\mathrm{CBM}=$ Concentração Bactericida Mínima; $\mathrm{CIM}_{50}=$ Concentração Inibitória Mínima capaz de inibir

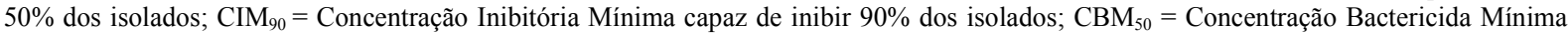
para 50\% dos isolados; $\mathrm{CBM}_{90}=$ Concentração Bactericida Mínima para $90 \%$ dos isolados; $\mathrm{MG}=$ Média Geométrica, em que letras iguais na mesma coluna indicam atividade antimicrobiana similar; OES = Óleos Essenciais, $O v=$ Origanum vulgare; $L g=$ Lippia graveolens; $T v=$ Thymus vulgaris; $C z=$ Cinnamomum zeylanicum. Nas comparações entre as CIMs e CBMs, utilizou-se o teste de Mann - Whitney $(\mathrm{P}<0,05)$ 
Tabela 2 - Relações entre o perfil de resistência de $\boldsymbol{E}$. coli a antimicrobianos e a susceptibilidade a óleos essenciais.

\begin{tabular}{|c|c|c|c|c|c|c|}
\hline & \multirow{2}{*}{ Subgrupo (n) } & \multirow{2}{*}{ Perfil de resistência } & \multicolumn{4}{|c|}{---1--1 } \\
\hline & & & Ov & $L g$ & $T v$ & $C z$ \\
\hline \multirow{16}{*}{ 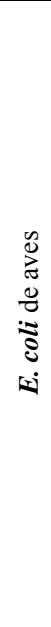 } & $1(16)$ & não resistente & $400-800$ & $800-3200$ & $1600-3200$ & 3200 \\
\hline & $2(3)$ & DOX & $400-800$ & $1600-3200$ & $1600-3200$ & 3200 \\
\hline & $3(4)$ & $\mathrm{AMO}$ & $400-1600$ & $1600-3200$ & 1600 & $1600-6400$ \\
\hline & $4(1)$ & EST & 400 & 1600 & 1600 & 3200 \\
\hline & $5(3)$ & DOX-EST & $400-1600$ & $1600-3200$ & $1600-3200$ & $1600-3200$ \\
\hline & $6(5)$ & EST-AMO & $400-1600$ & $1600-3200$ & $1600-3200$ & 3200 \\
\hline & $7(1)$ & SUT-EST & 1600 & 1600 & 1600 & 3200 \\
\hline & $8(1)$ & AMP-AMO & 800 & 3200 & 1600 & 3200 \\
\hline & $9(1)$ & DOX-AMOX & 1600 & 3200 & 3200 & 3200 \\
\hline & $10(2)$ & AMP-EST-AMO & 800 & $800-3200$ & 1600 & 3200 \\
\hline & $11(1)$ & AMC-EST- AMO & 800 & 1600 & 1600 & 3200 \\
\hline & $12(1)$ & DOX -EST-AMO & 400 & 1600 & 3200 & 3200 \\
\hline & $13(1)$ & DOX-AMC-AMO & 400 & 1600 & 3200 & 3200 \\
\hline & $14(1)$ & AMC-EST-GEN & 1600 & 1600 & 1600 & 3200 \\
\hline & $15(1)$ & CTF-NAL-EST-AMO & 1600 & 3200 & 3200 & 3200 \\
\hline & $16(1)$ & SUT-DOX-EST-GEN & 800 & 1600 & 1600 & 3200 \\
\hline \multirow{11}{*}{ 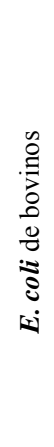 } & $1(9)$ & não resistente & $400-1600$ & $800-1600$ & $1600-6400$ & $3200-6400$ \\
\hline & $2(14)$ & AMO & $400-1600$ & $800-1600$ & $1600-6400$ & $3200-6400$ \\
\hline & $3(1)$ & DOX & 800 & 1600 & 3200 & 3200 \\
\hline & $4(4)$ & EST & 800 & $800-1600$ & $1600-3200$ & $3200-6400$ \\
\hline & $5(1)$ & EST-AMO & 800 & 800 & 1600 & 3200 \\
\hline & $6(1)$ & NAL-EST & 1600 & 800 & 3200 & 3200 \\
\hline & $7(1)$ & AMC-AMO & 800 & 1600 & 1600 & 6400 \\
\hline & $8(2)$ & NAL-AMO & $400-800$ & 1600 & 1600 & $3200-6400$ \\
\hline & $9(3)$ & AMC-EST-AMO & 1600 & 800 & 1600 & 3200 \\
\hline & $10(5)$ & SUT-DOX-AMC-EST-AMO & 800 & 800 & 1600 & 3200 \\
\hline & $11(5)$ & AMP-DOX-AMC-EST-AMO & 1600 & 1600 & 3200 & 3200 \\
\hline
\end{tabular}

AMP = ampicilina ; SUT = sulfametoxazol+trimetoprim; GEN = gentamicina; EST= estreptomicina; NAL = ácido nalidixico ; AMO = amoxicilina; $\mathrm{AMC}$ = amoxicilina + ácido clavulânico; CTF = ceftiofur; DOX = doxiciclina; CIMs = Concentrações Inibitórias Mínimas; (n) número de isolados; Ov = Oreganum vulgare; $\mathrm{Lg}=$ Lippia graveolens; $\mathrm{TV}=$ Thymus vulgaris.

promotores de crescimento (HUGHES et al., 2008). No Brasil, há crescente pressão pelas mesmas restrições, por isso alternativas substitutivas aos antimicrobianos tradicionalmente utilizados estão sob investigação (BRUGALLI, 2003).

As propriedades antimicrobianas dos óleos essenciais de plantas aromáticas são reconhecidas desde a Antiguidade (SIMÕES \& SPITZER, 2000). Neste estudo, avaliou-se a atividade antibacteriana de OES, obtidos de plantas condimentares sobre $\boldsymbol{E}$. coli patogênica para aves e bovinos. Os OES de orégano, orégano mexicano, tomilho e canela evidenciaram atividade antimicrobiana frente a $\boldsymbol{E}$. coli com variado perfil de susceptibilidade a antibacterianos.

A destacada atividade antimicrobiana do $\boldsymbol{O}$. vulgare frente a $\boldsymbol{E}$. coli de várias origens confirmou estudos anterioes. SMITH-PALMER et al. (1998) e HÖFERL et al. (2009) relataram importante atividade antimicrobiana do OE de orégano frente a bactérias Gram positivas e Gram negativas, incluindo E. coli. BURT et al. (2003) observaram que os óleos essenciais de orégano e tomilho possuem significativa atividade bacteriostática e bactericida frente $\boldsymbol{E}$. coli O157:H7, isoladas de fezes de bovinos. Os compostos fenólicos carvacrol e timol, enquanto frações majoritárias, garantem tais atividades. Essa atividade também tem sido relatada frente a fungos leveduriformes sensíveis e resistentes aos antimicóticos (POZZATTI et al., 2010).

A Lippia graveolens (orégano mexicano ou lipia) é uma planta condimentar originária do México. A composição de seu $\mathrm{OE}$ exibe significativos teores de carvacrol $(0,47-24,84 \%)$ e timol $(0,22-60,6 \%)$ (SÁNCHEZ 
et al., 2010). Vários estudos já evidenciaram atividade antimicrobiana $\left(\mathrm{CIM}=2000 \mu \mathrm{g} \mathrm{ml}^{-1}\right)$ sobre $\boldsymbol{E}$. coli ATCC 11229 (SÁNCHEZ et al., 2010); todavia, no presente estudo, detectou-se melhor potencial antimicrobiano MG das CIMs $=1508,7 \mu \mathrm{g} \mathrm{ml}^{-1}(\boldsymbol{E}$. coli de aves) e MG das CIMs $=1131,4 \mu \mathrm{g} \mathrm{ml}^{-1}$ (E. coli de bovinos) que o relatado por SANCHEZ et al. (2010).

Com base na composição média quantitativa das frações majoritárias carvacrol e timol nos OES de orégano (carvacrol 66\%; timol 1,0\%) (HÖFERL et al., 2009 ) lipia (carvacrol 38\%; timol 3.0\%) (SANCHES et al., 2010) e tomilho (carvacrol $<4.1 \%$ e timol $43.4 \%$ ) (HÖFERL et al., 2009). Os resultados encontrados neste trabalho evidenciaram que a menor concentração de carvacrol teve impacto decrescente na atividade antimicrobiana, o que torna-se claro nos resultados obtidos com o OE de tomilho.

No presente estudo, o OE de canela mostrou-se menos ativo que o de orégano, lipia e tomilho. SANTURIO et al. (2007), avaliando amostras de Salmonella enterica isoladas de aves e POZZATTI et al. (2010) estudando amostras de Candida spp., também constataram este perfil de ativade para o $\mathrm{OE}$ de canela. JAYAWARDENA \& SMITH (2010) avaliaram a composição do OES de Cinnamomum zeylanicum extraído de folhas no qual predominava o eugenol (98\%); já, quando extraído da casca, era rico em cinamaldeído (>80\%). HÖFERL et al. (2009) relataram fraca atividade do OE de folhas da canela (eugenol = 74\%) frente a $\boldsymbol{E}$. coli. Em contrapartida, BASKARAN et al. (2009) ressaltaram a fração cinamaldeído como mais ativa do que carvacrol, eugenol e timol. Assim, a atividade do OE de canela é dependente da parte do vegetal do qual é extraído, o que pode justificar as discrepâncias acima apontadas.

Frente aos OES de alecrim, sálvia, manjericão e gengibre, os resultados aqui apresentados não evidenciaram atividade antimicrobiana. Todavia, VIUDA-MARTOS et al. (2008) e DELAMARE et al. (2007) relataram atividade antimicrobiana dos OES de sálvia e alecrim frente a Staphylococcus spp, Enterobacter gergoviae, E. amnigenus, Lactobacillus sakei, e L. curvatus. A disparidade entre os resultados encontrados neste trabalho e os relatados por esses autores pode ser atribuída à composição dos OES ou às técnicas empregadas. Neste estudo, foi utilizada a técnica de microdiluição em caldo, com base no documento M31-A3 do CLSI (2008) para determinação das CIMs e CBMs. A inexistência de uma técnica internacionalmente padronizada para avaliação de OES e extratos vegetais permite que diferentes protocolos sejam utilizados, o que compromete as comparações de resultados VIUDA-MARTOS et al. (2008) e SMITHPALMER et al. (1998).
No presente estudo, a atividade dos OES sobre os subgrupos de $\boldsymbol{E}$. coli com diferentes perfis de resistência foi independente do maior ou menor padrão de resistência aos antibacterianos, o que está de acordo com outros autores. SI et al. (2008) relataram a atividade do OE de orégano sobre $\boldsymbol{E}$. coli ESBL (extendedspectrum $\beta$-lactamase) resistentes a múltiplos antibacterianos; MAHBOUBI \& BIDGOLI (2009) relataram que o $\mathrm{OE}$ de Zataria multiflora (condimento iraniano semelhante ao tomilho) composto por timol (38\%), carvacrol $(15,3 \%)$ e p-cimene (10,2\%) apresentou atividade antimicrobiana similar frente a isolados de Staphylococcus aureus meticilina resistente (MRSA) e sensíveis (MSSA). Frente a Candida, sensíveis e resistentes ao fluconazol. POZZATTI et al. (2010) relataram atividade antifúngica dos OES independentemente do perfil de resistência dos microrganismos.

O grande número de isolados e a variada procedência garantiram a heterogeneidade das amostras de E. coli para melhor avaliar a atividade antimicrobiana destes OES. A constatação de que a atividade dos OES de orégano, orégano mexicano, tomilho e canela é independente da susceptibilidade aos antibacterianos testados reforça a condição desses OES como candidatos a substituir antibacterianos, usados na alimentação de aves e bovinos.

\section{CONCLUSÃO}

Este estudo permitiu concluir que: a) os óleos essenciais de orégano, orégano mexicano, tomilho e canela apresentaram atividade bactericida frente a $\boldsymbol{E}$. coli isolados de aves e bovinos; b) o óleo essencial de orégano apresentou atividade antimicrobiana (CIMs e CBMs) superiores aos óleos essenciais de orégano mexicano, tomilho e canela; c) a susceptibilidade aos óleos essenciais foi semelhante entre subgrupos de isolados, independente da resistência aos antimicrobianos testados.

\section{AGRADECIMENTOS}

Os autores agradecem ao Conselho Nacional de Desenvolvimento Científico e Tecnológico (CNPq), pelo apoio financeiro.

\section{REFERÊNCIAS}

BASKARAN, A.S. et al. Antibacterial effect of plant-derived antimicrobials on major bacterial mastitis pathogens in vitro. Journal of Dairy Science, v.92, n.4, p.1423-1429, 2009. Disponível em: <http://download.journals.elsevierhealth.com/ pdfs/journals/0022-0302/PIIS0022030209704539.pdf $>$. Acesso em: 28 jan. 2011. doi: 10.3168/jds.2008-1384. 
BRUGALLI, I. Alimentação alternativa: a utilização de fitoterápicos ou nutracêuticos como moduladores da imunidade e desempenho animal. In: SIMPÓSIO SOBRE MANEJO E NUTRIÇÃO DE AVES E SUÍNOS, 2003, Campinas, SP. Anais... Campinas: Colégio Brasileiro de Nutrição Animal, 2003. V.1, p.167-182.

BURT, S.A., REINDERS, R.D. Antibacterial activity of selected plant essential oils against Escherichia coli O157:H7. Letters in Applied Microbiology v.36, p.162-167, 2003. Disponível em: <http://onlinelibrary.wiley.com/doi/10.1046/j.1472$65 \mathrm{X} .2003 .01285 . \mathrm{x} / \mathrm{pdf}>$. Acesso em: 28 jan. 2011. doi: 10.1046/j.1472-765X.2003.01285.x.

CLINICAL AND LABORATORY STANDARDS INSTITUTE (CLSI). Antimicrobial disk and dilution susceptibility tests for bacteria isolated from animals; approved Standard - 3.ed. Wayne,PA, 2008. (CLSI document M31-A3 Clinical and Laboratory Standards Institute).

DELAMARE, A.P.L. et al. Antibacterial activity of the essential oils of Salvia oficinalis $\mathbf{L}$. and Salvia triloba $\mathbf{L}$. cultivated in South Brazil. Food Chemistry, v.100, p.603-608, 2007. Disponível em: <http://dx.doi.org/10.1016/j.foodchem.2005.09.078>. Acesso em: 28 jan. 2011. doi: 10.1016/j.foodchem.2005.09.078.

GRIFFIN, P.; TAUXE, R.V. The epidemiology of infections caused by Escherichia coli O157:H7, other enterohemorrhagic Escherichia coli, and the associated hemolytic uremic syndrome. Epidemiologic Review v.13, p.60-98, 1991. Disponível em: $<$ http://epirev.oxfordjournals.org/content/13/1/60.long $>$. Acesso em: 28 jan. 2011.

HÖFERL, M. et al. Correlation of antimicrobial activities of various essential oils and their main aromatic volatile constituents. Journal of Essenntial Oil Research, v.21, p.459-464, 2009. Disponível em $<$ http://media.web.britannica.com/ebsco/pdf/020/ 44602020.pdf>. Acesso em: 28 jan. 2011.

HUGHES, L. et al. Risk factors for the use of prescription antibiotics on UK broiler farms. Journal of Antimicrobial Chemotherapy, v.61, p.947-952, 2008. Disponível em: $<$ http://jac.oxfordjournals.org/content/61/4/947.long $>$. Acesso em: 27 jan. 2011 . doi:10.1093/jac/dkn017.

ITO, N.M.K. et al. Diagnóstico diferencial das enfermidades bacterianas, fúngicas e parasitárias que acometem os frangos de corte. Cascavel, PR: Coluna do Saber, 2007. 160p.

JAYAWARDENA; B.; SMITH, R.M. Superheated water extraction of essential oils from Cinnamomum zeylanicum (L.). Phytochemical Analysis, v.21, p.470-472, 2010. Disponível em: <http://onlinelibrary.wiley.com/doi/10.1002/ pca.1221/abstract>. Acesso em: 28 jan. 2011. doi: 10.1002/ pca. 1221 .

LAMBERT, R.J.W. et al. A study of the minimum inhibitory concentration and mode of action of oregano essential oil, thymol and carvacrol. Journal of Applied Microbiology, v.91, p.453462, 2001. Disponível em: <http://onlinelibrary.wiley.com/doi/ 10.1046/j.1365-2672.2001.01428.x/abstract>. Acesso em: 28 jan. 2011. doi: 10.1046/j.1365-2672.2001.01428.x.

McMULLIN, P. Produção avícola após redução do uso de antibióticos. Riscos, potencial de contaminação cruzada e detecção de resíduos. North Yorkshire, U.K., 2004. In: CONFERÊNCIA APINCO, 2., 2004, Campinas SP. Anais... Campinas: FACTA, 2004. V.2. 291p. p.219.
MAHBOUBI, M.; BIDGOLI, F.G. Antistaphylococcal activity of Zataria multiflora essential oil and its synergy with vancomycin. Phytomedicine, v.17, p.548-550, 2010. Disponível em: <http:/ /dx.doi.org/10.1016/j.phymed.2009.11.004>. Acesso em: 28 jan. 2011. doi: 10.1016/j.phymed.2009.11.004.

POZZATTI, P. et al. Comparison of the susceptibilities of clinical isolates of Candida albicans and Candida dubliniensis to essential oils. Mycoses, v.53, p.12-15, 2010. Disponível em: <http://onlinelibrary.wiley.com/doi/10.1111/j.14390507.2008.01643.x/abstract>. Acesso em: 20 jan. 2011. doi: $10.1111 /$ j.1439-0507.2008.01643.x.

SÁNCHEZ, A.A. et al. Antimicrobial and antioxidant activities of Mexican oregano essential oils (Lippia graveolens H. B. K.) with different composition when microencapsulated in $B$ cyclodextrin. Letters in Applied Microbiology, v.50, p.585590, 2010. Disponível em: <http://onlinelibrary.wiley.com/ doi/10.1111/j.1472-765X.2010.02837.x/abstract>. Acesso em: 20 jan. 2011. doi:10.1111/j.1472-765X.2010.02837.x

SANTURIO, J. et al. Atividade antimicrobiana dos óleos essenciais de orégano, tomilho e canela frente a sorovares de Salmonella enterica de origem avícola. Ciência Rural, v.37, n.3, p.803-808, 2007. Disponível em: <http://www.scielo.br/ pdf/cr/v37n3/a31v37n3.pdf>. Acesso em: 28 de jan. 2011.

SARIDAKIS, H.O. et al. Virulence properties of Escherichia coli strains belonging to enteropathogenic (EPEC) serogroups isolated from calves with diarrhea. Veterinary Microbiology, v.54, p.145-153, 1997. Disponível em <http://dx.doi.org/ 10.1016/S0378-1135(96)01278-3>. Acesso em: 28 jan. 2011. doi:10.1016/S0378-1135(96)01278-3.

SI, H. et al. Antibacterial effect of oregano essential oil alone and in combination with antibiotics against extended-spectrum ß-lactamase-producing Escherichia coli. FEMS Immunol Med Microbiol, v.53, p.190-194, 2008. Disponível em: <http:// onlinelibrary.wiley.com/doi/10.1111/j. 1574 695X.2008.00414.X/abstract>. Acesso em: 25 jan. 2011. doi: $10.1111 /$ j.1574-695X.2008.00414.x.

SIMÕES, C.M.O.; SPITZER, V. Óleos voláteis. In: SIMÕES, C.M.O. et al. Farmacognosia: da planta ao medicamento. Porto Alegre/Florianópolis: UFRGS/UFSC, 2000. Cap.18.

SMITH-PALMER, A. et al. Antimicrobial properties of plant essential oils and essences against five important food-borne pathogens. Letters in Applied Microbiology, v.26, p.118122, 1998. Disponível em: < http://onlinelibrary.wiley.com/ doi/10.1046/j.1472-765X.1998.00303.x/abstract>. Acesso em: 28 jan. 2011 . doi: 10.1046/j.1472-765X.1998.00303.x.

VIUDA-MARTOS, M. et al. Antibacterial activity of different essential oils obtained from spices widely used in Mediterranean diet. International Journal of Food Science and Technology, v.43, p.526-531, 2008. Disponível em: <http:// onlinelibrary.wiley.com/doi/10.1111/j. 1750 3841.2008.00966.x/abstract>. Acesso em: 28 jan. 2011. doi: $10.1111 /$ j.1750-3841.2008.00966.x.

WORLD HEALTH ORGANIZATION (WHO). Joint FAO/ OIE/WHO EXPERT WORKSHOP ON Non-HUMAN ANTIMICROBIAL USAGE AND ANTIMICROBIAL RESISTANCE: SCIENTIFIC ASSESSMENT," Presented in Geneva, Switzerland, Dec. 2003. Disponível em: < www.who.int/ foodsafety/micro/meetings/nov2003/en/>. Online. Acesso em: 24 nov. 2010. 